\title{
Statistical media optimization studies for growth and polydroxybutyrate (PHB) production by Pseudomonas spp.
}

\author{
Mukesh R. Jangra*, Ritu Batra, Ikbal, Akanksha Jain, Rekha Ahlawat and Virendra K. Sikka
}

Department of Molecular Biology, Biotechnology \& Bioinformatics, College of Basic Sciences \& Humanities, Chaudhary Charan Singh Haryana Agricultural University, Hisar-125004 (Haryana), INDIA

*Corresponding author. E-mail: jangra.mukesh02@gmail.com

Received: October 20, 2015; Revised received: April 27, 2016; Accepted: July 18, 2016

Abstract: Using glucose as carbon source and mustard cake and yeast extract as nitrogen sources bacterial isolate Pseudomonas B2 exhibited a maximum PHB recovery of 0.620 (in terms of O.D.) and PHB weight of $0.27 \mathrm{~g} / \mathrm{L}$ in 96 h. To determine the possibility of growth potential of Pseudomonas spp., it was grown in different carbon sources like fructose, glucose, maltose, mannitol etc. and it was found that glucose yielded good growth and PHB production. In order to incorporate cost effective nitrogen and carbon source, mustard cake and cotton cake as nitrogen source and molasses as carbon were used in medium. Statistical media optimization design was used to optimize the culture conditions for maximizing the PHB production. A maximum of $0.37 \mathrm{~g} / \mathrm{L}$ of PHB and 0.746 (O.D.) PHB recoveries were obtained using optimized concentrations. Batch kinetics can be used for model development, which will make possible simulation of nutrient limited cultivation(s) for over accumulation of PHB. FTIR studies confirmed the presence of PHB.

Keywords: Central composite design, Pseudomonas spp., Polyhydroxybutyrate, Response Surface Methodology

\section{INTRODUCTION}

Poly- $\beta$-hydroxybutyric acid (PHB) is a compostable, natural and biodegradable polymer, which is accumulated under stress conditions in the form of intracellular granules by diverse microorganisms. These granules take action as energy reserve materials when there is nutrients depletion like nitrogen and phosphorous source in the presence of excess carbon source (Byrom, 1987; Anderson et al, 1990). They can be completely degraded by microorganisms and form water and carbon dioxide and methane under aerobic conditions and anaerobic conditions respectively in soil, sea, lake water and sewage (Lee, 1996). The main hindrance in the large-scale production and commercialization of PHB is their high cost and time of production as compared to petroleum based plastics. One of the major factors responsible for the cost of PHB is the expenditure of substrates used for production. So, cheaper substrates, superior cultivation strategies and easier downstream processing methods are essential for reducing the cost (Ahn et al., 2001; Jangra et al., 2016). Thus, utilization of media containing low cost carbon and nitrogen sources like molasses, potato peels, mustard cake and cotton cake should be used to trim down the production costs of PHB (Jangra and Sikka, 2015). Members of Pseudomonas sp., Aeromonas hydrophila and Bacillus sp. accumulate heteropolymers of short chain length- medium chain length PHAs (scl-mclPHA) such as P(3HB-co- 3HV), P(3HB
-co-3HHx) and $\mathrm{P}(3 \mathrm{HB}-\mathrm{co}-4 \mathrm{HB})$ when substrates like c -butyrolactone or e-caprolactone are present as carbon source in the growth medium (Labuzeck and Radecka 2001). Optimization of culture conditions has long been used to get better yield and productivities of many bioprocesses. Conventionally, optimization of media was very time consuming. Culture conditions were optimized by doing the variation of one component or parameter at a time, but the process variables do not interrelate and that process response is function of a single factor, which is varied. But now a day's statistical optimization methods replaced these conventional approaches by studying the interaction of variables in generating the response and provide $3 \mathrm{D}$ plots for the interactions (Veeramanikandan et al, 2013). Central Composite Design of response surface methodology is one of the computer based statistical designs for the selection of the independent variables. Response surface methodology (RSM) is a wellorganized tool used to study the interactive effect of parameters take part in fermentation process in search of optimized conditions for enhanced and improved product yields (Montgomery 2005; Jangra et al., 2016). In the present study, bacterial growth was initially carried out in a minimal medium. Thereafter, different carbon and nitrogen sources with combinations of agri byproducts were used to analyze their effects on growth and PHB production. By taking the best and low cost carbon and nitrogen source, statistical media optimization was carried out by the experi- 
ments designed using response surface methodology. Optimized media was finally used to study the kinetics of growth under constant $\mathrm{pH}$ conditions to investigate the improvement in growth in terms of optical density and PHB recovery by bacterial strain Pseudomonas B2.

\section{MATERIALS AND METHODS}

Microorganism: Pseudomonas B2 isolated from oil spilled soil, Hisar was used in all experiments. The culture was maintained on nutrient agar slants at $37^{\circ} \mathrm{C}$, and sub cultured monthly.

Media For initial study: A mineral salt medium which consisted of: $2 \mathrm{~g} / \mathrm{l}$ Glucose, $0.5 \mathrm{~g} / 1 \mathrm{KNO}_{3}, 0.5 \mathrm{~g} / 1 \mathrm{~K}_{2} \mathrm{HPO}_{4}$ $0.2 \mathrm{~g} / \mathrm{l} \mathrm{MgSO}{ }_{4} .7 \mathrm{H}_{2} \mathrm{O}, 0.1 \mathrm{~g} / \mathrm{l} \mathrm{NaCl}, 0.06 \mathrm{~g} / \mathrm{l} \mathrm{CaCl} 2.2 \mathrm{H}_{2} \mathrm{O}$. Glucose and $\mathrm{KNO}_{3}$ were used as carbon and nitrogen source. MM (Minimal Media) (Beringer et al., 1978) was sterilized separately at $121^{\circ} \mathrm{C}$ and then aseptically reconstituted at room temperature before inoculation.

For comparison of different carbon and nitrogen sources: Same media (described above) was used for all the experiments. However, for comparison, different carbon (3-5 g/L) and nitrogen (2-4 g/L) sources were taken for comparison.

Methods Study of growth kinetics of the culture: Minimal salt medium containing $2 \mathrm{~g} / \mathrm{L}$ glucose was used for inoculums preparation. The organism was cultivated at stationary condition and $37^{\circ} \mathrm{C}$ for $24 \mathrm{hrs}$ in a $250 \mathrm{~mL}$ Erlenmeyer flask which contain $50 \mathrm{~mL}$ of the medium as described. Initial $\mathrm{pH}$ was constant. For production of $\mathrm{PHB}$, one litre of media (containing $2 \mathrm{~g} / \mathrm{L}$ glucose) was taken in a $2000 \mathrm{~mL}$ flask and inoculated with $50 \mathrm{~mL}$ of inoculum. The flask was kept under stationary conditions for $120 \mathrm{hrs}$ at $37^{\circ} \mathrm{C}$. Samples were withdrawn at regular intervals and analyzed for PHB recovery and PHB weight.

Effect of different carbon sources on PHB recovery and production: The $\mathrm{PHB}$ production was carried out using fructose, glucose, maltose, sorbitol, chitin, peptone, CMC, dextrose, glycerol, mannitol, starch, galactose, molasses. The production medium in each flask consisted of the minimal salt medium and a different carbon source $(3-5 \mathrm{~g} / \mathrm{L})$ with potassium nitrate as a common nitrogen source in all media compositions. The flasks were incubated under stationary conditions at $37^{\circ} \mathrm{C}$. PHB recovery and PHB weight were estimated in the culture broth after $96 \mathrm{~h}$. After $96 \mathrm{~h}$ of growth cells were harvested and centrifuged at $10,000 \mathrm{rpm}$ and cells pellet were further analyzed for PHB recovery.

Comparison of PHB production in different nitrogen sources: Different nitrogen sources investigated were: Ammonium Chloride, Ammonium Nitrate, Ammonium Acetate, Ammonium Sulphate, Cotton cake, Potassium Nitrate, Sodium azide, Mustard cake, Yeast extract and Urea. The production medium in each flask consisted of the minimal salt medium and different nitrogen sources (3-5 g/L) with glucose as common car- bon source and proceeded according to above protocol. Effect of different combinations of agri byproducts with organic and inorganic carbon and nitrogen sources on PHB production: Different combinations of agri-byproducts like molasses, mustard cake and cotton cake were used with inorganic and organic carbon and nitrogen sources for comparison of PHB production by crotonic acid estimation. It was found that strains B2 produced maximum PHB when carbon source glucose used in combination of two different nitrogen sources mustard cake and yeast extract.

Analytical methods: Determination of the amount of PHB was by the chemical method (Slepecky and Law, 1961). Cell mass was collected by centrifuging bacterial culture broth at $10,000 \mathrm{rpm}$. The cell pellet collected from culture broth was treated with $10 \mathrm{ml} \mathrm{so-}$ dium hypochlorite $(4 \%)$ by vortexing it and samples were boiled for five to ten minutes in boiling water bath. After cooling $20 \mathrm{ml}$ of chloroform was added to the boiled samples. Keep it at $65^{\circ} \mathrm{C}$. Chloroform evaporates and dissolved PHB recovered. $50 \mu \mathrm{l}$ of dissolved PHB added to $2 \mathrm{ml}$ of sulphuric acid and then incubated for one day at $65^{\circ} \mathrm{C}$. After that the spectrophotometer reading is taken at $235 \mathrm{~nm}$. The components giving higher PHB recovery and weight were selected for further studies needed for optimization.

Experimental design and optimization using response surface methodology: By using response surface methodology, the levels of the significant parameters and interaction effects between components of media and the bacterial strain viz., Pseudomonas B2 were analyzed and optimized. The experiment was designed using "Stat-Ease Design-Expert" software (Stat Ease, Inc Design Expert software, trial version 9.0.3, Minneapolis, USA). The three independent factors were investigated at five different levels. $(-2,-1$, $0,+1,+2)$. The response Y (PHB recovery) was studied and analyzed by using a second order polynomial equation in three independent variables and the data were fitted into the equation by multiple regression procedure. "Design-Expert" 9.0.3 was used for regression and graphical analyses of the data which was obtained from the experiments. Statistical analysis of the model was done to calculate the analysis of variance (ANOVA). The student's t-test and the Fischer's test permitted and help to the checking of the statistical significance of the regression coefficient and determine the second-order model equation respectively. The excellence of the fit of the polynomial model equation was verified and specified by the coefficient of determination (R2).

Analysis of agro-PHB for its organic components and physical properties : Physico chemical analysis of the eco-friendly agro-PHB and agro bioplastic developed above was done using FTIR and NMR spectroscopy.

Fourier-transform infrared spectroscopy (FTIR FTIR has been demonstrated to be a powerful 
tool for screening various types of PHAs. It is used to detect functional groups in an organic compound. FTIR spectra of PHB that was recorded in $\mathrm{KBr}$ (GJUS\&T, Hisar).

Nuclear Magnetic Resonance spectroscopic analysis of PHB: ${ }^{1} \mathrm{H}$ NMR spectroscopic analysis of PHB that was produced from strain B2 was done. For Nuclear Magnetic Resonance (NMR) spectroscopy, the polymer was suspended in spectrochem grade deuterochloroform $\left(\mathrm{CDCl}_{3}\right)$. The ${ }^{1} \mathrm{H}$ NMR spectra of sample was obtained at $300 \mathrm{MHz}$ IIT, Delhi. The chemical shift scale was in parts per million (ppm).

Poly hydroxyl butyrate miscibility analysis with polymer plastic and starch: The polyhydroxybutyrate extracted in chloroform was mixed with equal weight of poly-propylene polymer plastic. The mixture was incubated at $70^{\circ} \mathrm{C}$ for 10 minutes and then poured into a glass petri plate. A film of blended mixture with polymer plastic and starch was obtained.

Biodegradability Analysis of of AgriPHB/ bioplastic: Biodegradation process of agro bioplastic was also studied under lab conditions. For this purpose equal amount of agro-bioplastic was added to tubes containing sterile distilled water, soil dilutions, sewage water and water containing bacterial culture. The process of degradation was studied till thirty days. It was examined at weekly intervals to observe extent of degradation.

\section{RESULTS AND DISCUSSION}

Initial studies using MM media and growth kinetics for PHB: PHB Kinetics (Fig. 1) was studied in bacterial isolate using one litre of minimal medium and incubated at $37^{\circ} \mathrm{C}$. Samples were drawn at incremental time intervals spread over several days and the amount of PHB was extracted using chloroform and sodium hypochlorite method and quantified by crotonic acid estimation. As per the result shown in fig. 1, it was observed that strain B2 peaked to maximum $\mathrm{PHB}$ production in 96 $\mathrm{h}$ and PHB was expressed as Crotonic acid.

Comparison of different carbon sources for maximum PHB production: A comparative study was done using different carbon sources for maximum PHB production. It was found that molasses were the best carbon source (Fig. 2) which is cheaper than fructose and the next promising sources were glucose and

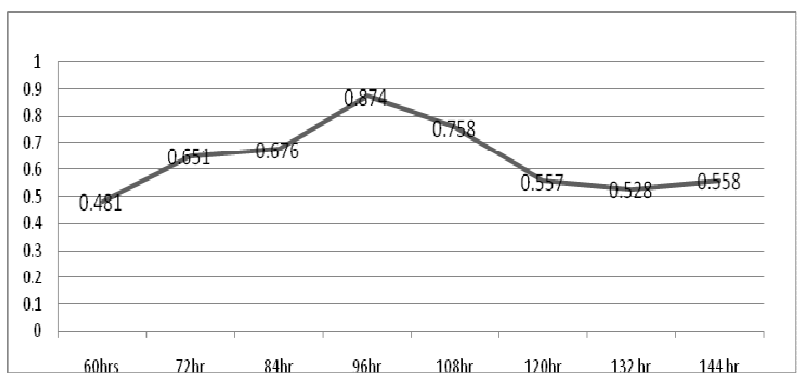

Fig. 1. Production kinetics of B2 strain expressed as Crotonic acid (O.D. 235). fructose (PHB expressed as Crotonic acid (O.D. 235). Molasses was used as a carbon source (sucrose and other carbohydrates) and it is very cost effective substrates which will help in reducing the cost of PHB production. But in reality, it is a byproduct in the sugar industry. We should remember that most of the industrial by-products (including molasses) contain ions and minerals that have deleterious effects on microbial growth and product synthesis. Apart from it, different cost-effective nitrogen sources like corn steep liquor, chick pea bran, soy bean meal, mustard cake etc. can be used for PHB production (Sharma and Bajaj, 2015). Effect of different nitrogen sources for maximum PHB production: Since nitrogen source is also an important factor for the accumulation of PHB, different salts of ammonium (Fig. 3) were tested to maximize the PHB production and it was found that potassium nitrate was the best nitrogen source and after this yeast extract, cotton cake, mustard cake can be the good nitrogen sources for the PHB production.

PHB production using defined carbon and organic nitrogen from agri-byproducts along with carbon from agribyproducts and inorganic nitrogen in B2 strain: To make the of PHB production protocol less costly, carbon and nitrogen sources were used in combination with agribyproducts (Table 1) and it was found that when glucose was used in combination with mustard cake and yeast extract, PHB production was maximum. Table 1 shows the cell pellet $(\mathrm{g} / \mathrm{L})$ PHB recovery (O.D.) and $\mathrm{PHB}$ weight obtained with the different combinations. A maximum cell pellet of 2.96 $\mathrm{g} / \mathrm{L}, \mathrm{PHB}$ recovery $1.375 \mathrm{~nm}$ and $\mathrm{PHB}$ weight $27 \mathrm{mg} / \mathrm{L}$ after 96 hrs was observed.So, on the basis of above results, three independent factors glucose, mustard cake and yeast extract and two responses PHB recovery and PHB weight were chosen for optimization studies. CCD of RSM was used for the optimization of the culture conditions to maximize the PHB production.

Response surface methodology: Response surface methodology is a successive, exploratory approach to establish the relationship between more than one variable with the obtained responses. The analysis develops a model by fitting the experimental data in a generalized smooth curve, from which a specific predicted

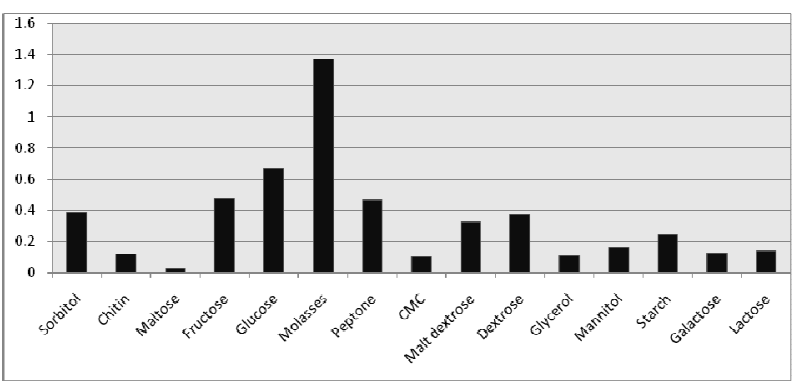

Fig.2 .Effect of different carbon source on PHB production. 
Mukesh R Jangra et al. / J. Appl. \& Nat. Sci. 8 (3): 1278 - 1285 (2016)

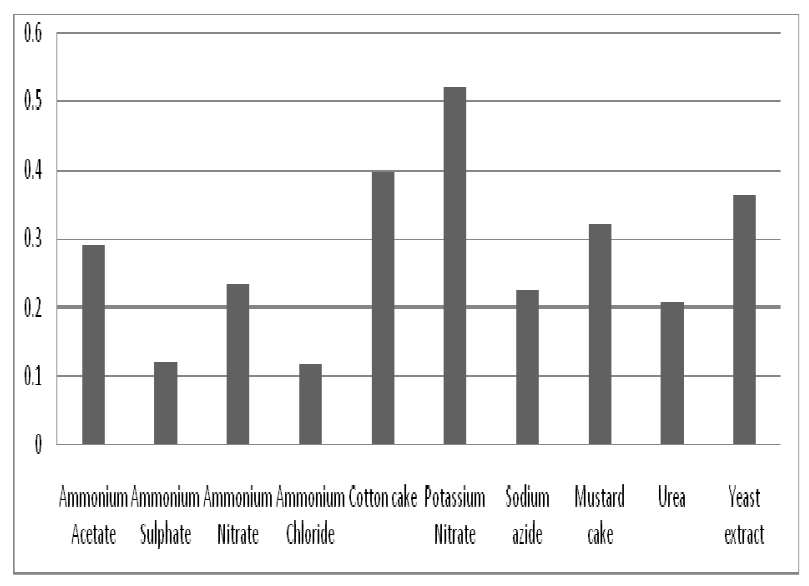

Fig. 3. Effect of different nitrogen source on PHB.

response could be calculated. In addition, 3D plots are also generated which delineates predicted response over a range in the design surface. Thus, a step-by-step approach for response surface analysis establishes a relation between variable and response more efficiently than traditional design (Launen et al., 1999).
Based on the central composite design, three factors (glucose as carbon source and mustard cake and yeast extract) that showed positive influence on PHB recovery and PHB weight were selected and CCD (Central Composite Design) was used to determine the optimum levels of these parameters. A total of 20 experiments with different combinations of glucose (A), mustard cake (B) and yeast extract (C) were performed (Table 2). The results were analyzed by Design Expert Software and following quadratic regression equations were obtained in terms of the selected variables for growth and PHB production:

Optical density of $\mathrm{B2}$ strain $(\mathbf{n m})(\mathrm{Y} 1)=+0.66$ $+0.037 * \mathrm{~A}+0.090 * \mathrm{~B}-0.011 * \mathrm{C}-0.019 * \mathrm{AB}+0.030 * \mathrm{AC}$ $-0.015 * \mathrm{BC}+0.025 * \mathrm{~A}^{\wedge} 2-0.035^{*} \mathrm{~B}^{\wedge} 2-0.013 * \mathrm{C}^{\wedge} 2(1)$

PHB recovery of $\mathrm{B} 2$ strain $(\mathrm{g} / \mathrm{l})(\mathrm{Y} 2)=+0.033$ $+3.365 \mathrm{E}-003 * \mathrm{~A} \quad+7.684 \mathrm{E}-003 * \mathrm{~B} \quad-1.471 \mathrm{E}-003 * \mathrm{C}-$ $2.250 \mathrm{E}-003 * \mathrm{AB}+7.500-004 * \mathrm{AC}-7.500 \mathrm{E}-004 * \mathrm{BC}-$ $1.600 \mathrm{E}-003 * \mathrm{~A}^{\wedge} 2-4.605 \mathrm{E}-003 * \mathrm{~B}^{\wedge} 2-2.838 \mathrm{E}-003 * \mathrm{C}^{\wedge}$ (2)For determination of the optimum operating concentration and interaction of factors on PHB recovery

Table 1. PHB production using different combinations (Average of three independent experiments)

\begin{tabular}{|c|c|c|c|c|c|}
\hline S. $\mathbf{N}$. & $\begin{array}{c}\text { Carbon } \\
\text { source }\end{array}$ & Nitrogen source & Pellet wt.( g/l) & PHB recovery(O.D.) & $\begin{array}{c}\text { PHB weight (mg/ } \\
\text { L) }\end{array}$ \\
\hline 1. & Molasses & Mustard cake+Yeast Extract & 1.614 & 0.629 & 12 \\
\hline 2. & Molasses & Cotton cake+Yeast Extract & 1.965 & 0.357 & 2 \\
\hline 3. & Molasses & Cotton cake+ $\mathrm{KNO}_{3}$ & 1.643 & 0.563 & 7 \\
\hline 4. & Molasses & Cotton cake & 1.312 & 0.494 & 6 \\
\hline 5. & Glucose & Mustard cake+ $\mathrm{KNO}_{3}$ & 1.478 & 0.853 & 8 \\
\hline 6. & Glucose & Cotton cake+ $\mathrm{KNO}_{3}$ & 2.851 & 1.095 & 25 \\
\hline 7. & Glucose & Mustard cake+ Yeast Extract & 2.967 & 1.375 & 27 \\
\hline 8. & Glucose & Cotton cake+ Yeast Extract & 2.195 & 0.796 & 22 \\
\hline 9. & Fructose & Cotton cake+ Yeast Extract & 2.225 & 0.762 & 20 \\
\hline 10. & Fructose & Mustard cake+ Yeast Extract & 1.410 & 0.959 & 26 \\
\hline
\end{tabular}

Table 2.Response surface methodology yield of PHB by strain B2.

\begin{tabular}{|c|c|c|c|c|c|}
\hline & Factor 1 & Factor 2 & Factor 3 & Response 1 (Y1) & Response 2(Y2) \\
\hline $\begin{array}{c}\mathbf{R u} \\
\mathbf{n}\end{array}$ & $\begin{array}{l}\text { A:Glucose (C- } \\
\text { Source) }\end{array}$ & $\begin{array}{l}\text { B:Mustard Cake (N- } \\
\text { Source) }\end{array}$ & C:YE (N-Source) & O.D & PHB \\
\hline & $\mathrm{g} / \mathrm{l}$ & $\mathrm{g} / \mathrm{l}$ & $\mathrm{g} / \mathrm{l}$ & $\mathrm{nm}$ & $\mathrm{mg} / \mathrm{l}$ \\
\hline 1 & 4 & 0.5 & 0.4 & 0.554 & 21 \\
\hline 2 & 3 & 1 & 0.8 & 0.687 & 31 \\
\hline 3 & 3 & 1.840 & 0.8 & 0.746 & 37 \\
\hline 4 & 4.6 & 1 & 0.8 & 0.884 & 4 \\
\hline 5 & 2 & 1.5 & 1.2 & 0.659 & 27 \\
\hline 6 & 3 & 1 & 0.8 & 0.693 & 29 \\
\hline 7 & 3 & 1 & 0.8 & 0.667 & 32 \\
\hline 8 & 2 & 0.5 & 0.4 & 0.528 & 13 \\
\hline 9 & 2 & 0.5 & 1.2 & 0.523 & 12 \\
\hline 10 & 4 & 1.5 & 0.4 & 0.674 & 3 \\
\hline 11 & 3 & 1 & 0.8 & 0.681 & 3 \\
\hline 12 & 3 & 1 & 1.4 & 0.608 & 24 \\
\hline 13 & 4 & 1.5 & 1.2 & 0.73 & 29 \\
\hline 14 & 2 & 1.5 & 0.4 & 0.799 & 34 \\
\hline 15 & 3 & 1 & 0.12 & 0.672 & 3 \\
\hline 16 & 3 & 1 & 0.8 & 0.669 & 28 \\
\hline 17 & 1.3 & 1 & 0.8 & 0.608 & 21 \\
\hline 18 & 4 & 0.5 & 1.2 & 0.596 & 2 \\
\hline 19 & 3 & 0.15 & 0.8 & 0.407 & 7 \\
\hline 20 & 3 & 1 & 0.8 & 0.588 & 5 \\
\hline
\end{tabular}


Mukesh R Jangra et al. / J. Appl. \& Nat. Sci. 8 (3): 1278 - 1285 (2016)

Table 3. ANOVA Coefficient estimate for O.D. and PHB recovery.

\begin{tabular}{ccccc}
\hline & O.D. & O.D. & PHB recovery & PHB recovery \\
\cline { 2 - 4 } & F value & P-value & F value & P-value \\
\hline Model & 5.97 & 0.0050 & 3.62 & 0.0288 \\
A-Glucose (C Source) & 5.82 & 0.0366 & 3.51 & 0.0904 \\
B-Mustard Cake (NSource) & 34.01 & 0.0002 & 18.14 & 0.0017 \\
C-Yeast Extract (N-Source) & 0.54 & 0.4807 & 0.67 & 0.4318 \\
AB & 0.90 & 0.3660 & 0.92 & 0.3602 \\
AC & 2.26 & 0.1635 & 0.10 & 0.7558 \\
BC & 0.56 & 0.4712 & 0.10 & 0.7558 \\
$\mathrm{~A}^{\wedge} 2$ & 2.67 & 0.1336 & 0.84 & 0.3816 \\
$\mathrm{~B}^{\wedge} 2$ & 5.52 & 0.0407 & 6.94 & 0.0250 \\
$\mathrm{C}^{\wedge} 2$ & 0.74 & 0.4111 & 2.63 & 0.1356 \\
$\mathrm{R}^{2}$ & & 0.87 & & 0.91 \\
Lack of Fit & & 0.025 & & 0.028 \\
\hline
\end{tabular}
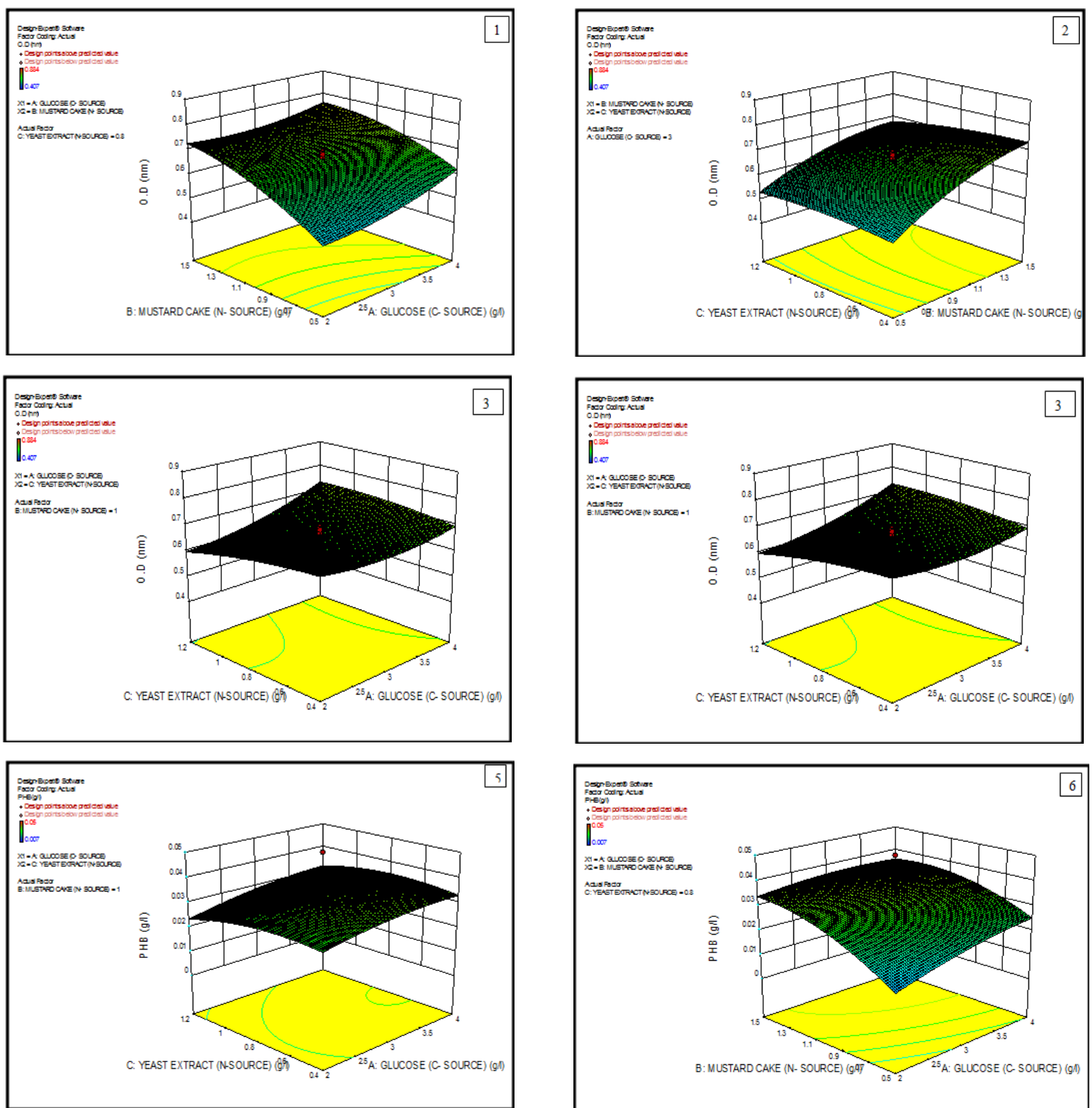

Fig.4. 3-D plots showing the interactive effect of mustard cake, yeast extract and glucose for the responses optical density and PHB recovery in strain $B 2$. 


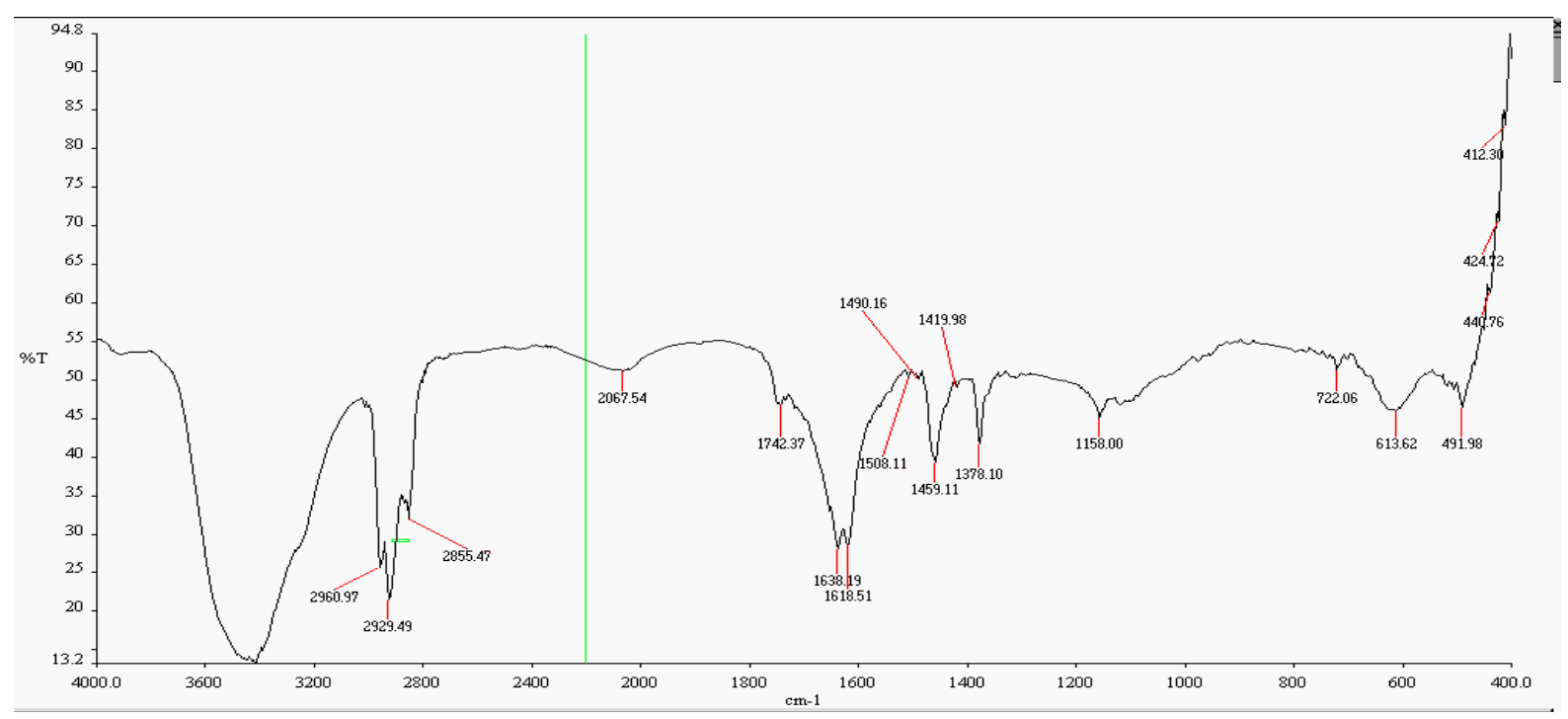

Fig 5. FTIR spectrum of $P H B$

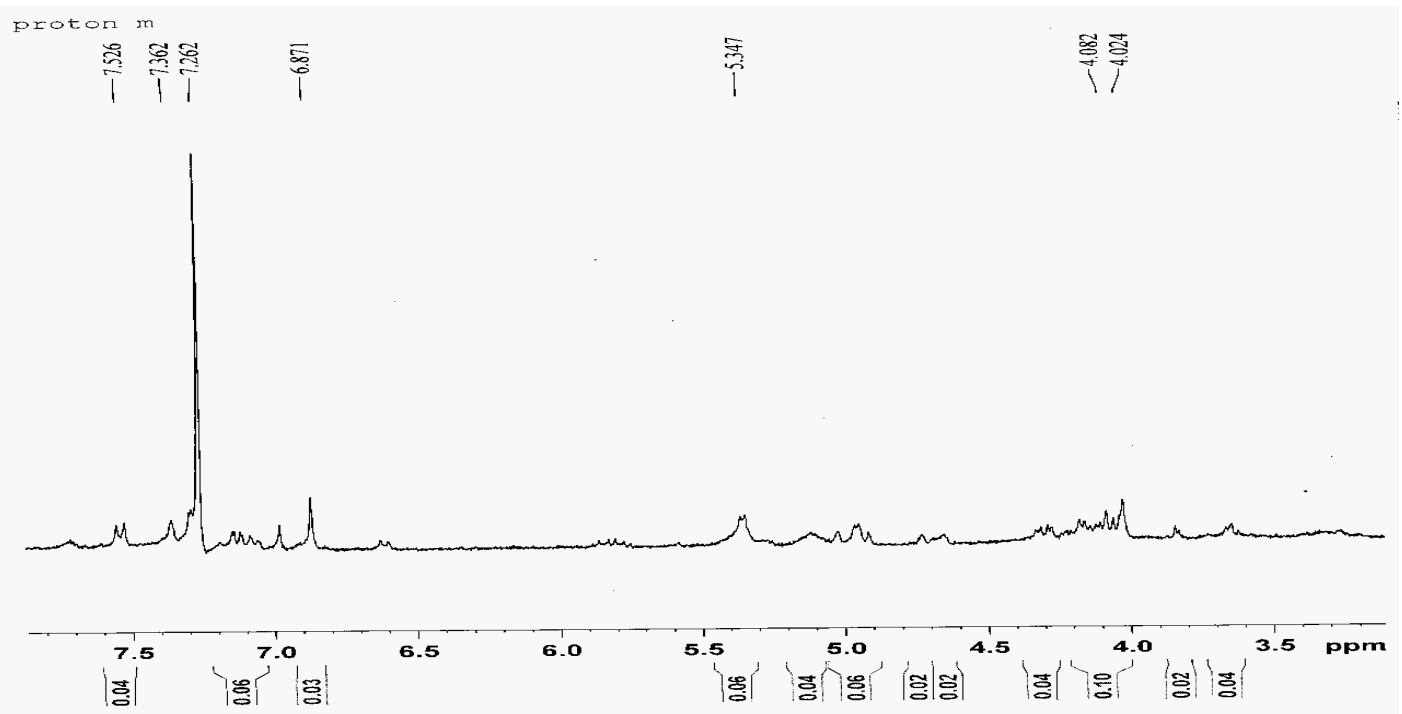

Fig 6. NMR spectra produced by $B 2$ strain

(O.D.) PHB weight, the response surfaces were studied in detail for all possible combinations by keeping three parameters constant at a time. The 3D (Fig. 4) between various factors was analyzed and optimized concentrations of the media components were found. The significance of coefficient of fitted quadratic model was evaluated by using F-value and P-value. The analysis of variance (ANOVA) for optical density and PHB of quadratic model eq. $(1,2)$ of B2 strain is given in (Table 3). The Model F-value of 5.97 for O.D. and 3.62 for PHB recovery implied the model was significant. Significance of model terms were indicated by values of "Prob > F" less than $0.0500 . \mathrm{A}, \mathrm{B}, \mathrm{B}^{\wedge} 2$ were significant model terms. Values greater than 0.1000 indicate the model terms were not significant. Many insignificant model terms leads to model reduction which may improve the model. The "Lack of Fit Fvalue" was 0.025 for O.D. (Crotonic acid estimation) \& 0.28 for PHB recovery implied the Lack of Fit was not significant relative to the pure error. Nonsignificant lack of fit was good for the fitness of model. The fit of model was also expressed by the coefficient of determination R2, which was found to be 0.87 for O.D. and 0.91 for PHB recovery. The analysis of variance for B2 strain (Tables 3) indicated that B, $B^{\wedge} 2$ as significant terms $(p<0.05)$ for cell optical density and PHB recovery. The sign and magnitude of the coefficients indicated the effect of the variables on the responses. Fig. 4 (1-6) shown the surface plots for the interactive factors glucose, mustard cake and yeast extract. The $3 \mathrm{D}$ response surface plots were generally the graphical representation of regression equation and 

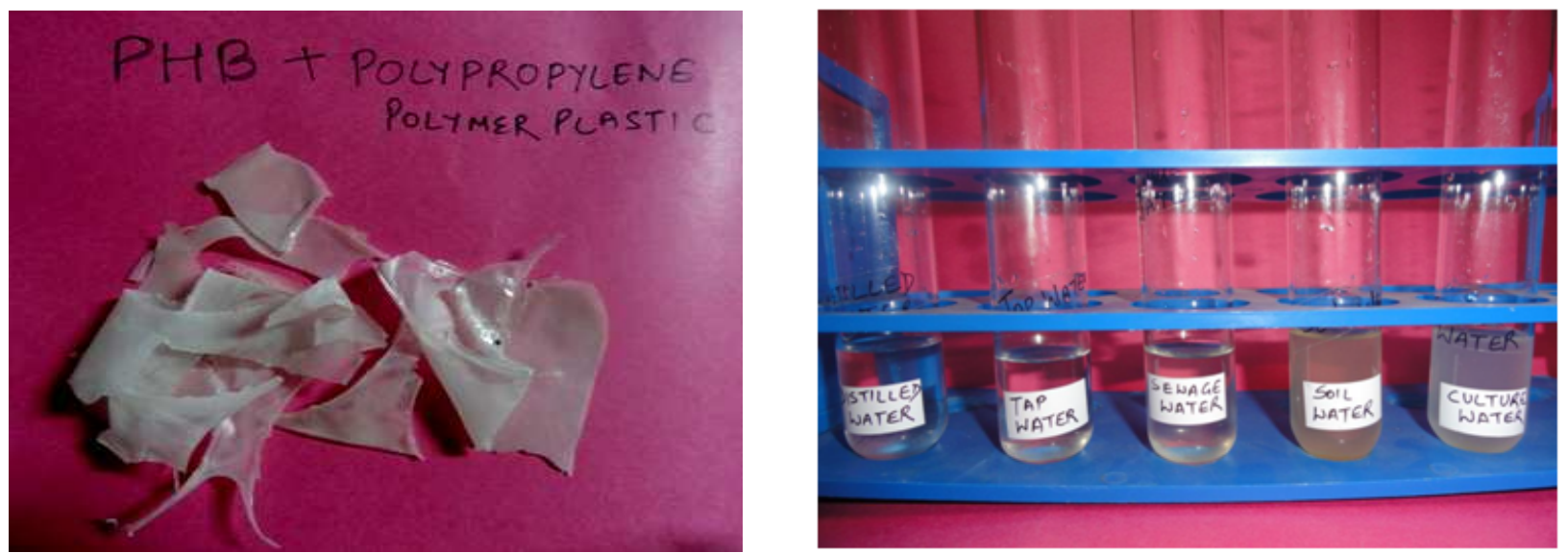

Fig 7. A) A film of blended mixture polyhydroxybutyrate and poly-propylene polymer plastic, B) Biodegradability Analysis of of Agribioplastic.

the interaction between variables could be inferred from the shapes of the surface plots. The maximum predicted optical density (0.884) increased with increase of glucose and mustard cake up to $4.6 \mathrm{~g} / \mathrm{l}$ and $1 \mathrm{~g} / \mathrm{l}$ and with decrease of yeast extract (Fig. 4, 1-3). PHB recovery (Fig. 4, 4-6) also increased with increase of glucose and mustard cake up to $3 \mathrm{~g} / \mathrm{l}$ and $1.8 \mathrm{~g} / \mathrm{l}$. Results indicated that responses varied much as function of concentration of glucose and mustard cake. The graphs showing the interaction between carbon source glucose, nitrogen sources mustard cake and yeast extract have been presented in fig. 4(1-6). So it was essential to maintain proper $\mathrm{C} / \mathrm{N}$ ratio in growth associated PHB producing bacteria to achieve maximum PHB production. Similar results were observed by Jangra et al. (2016).

FTIR and NMR spectrum analysis: Fourier Transform Infra-Red (FTIR) spectrum of the PHB sample (Fig. 5) revealed large peak nearly at 2956.97 which represents the $-\mathrm{CH} 2$ groups, followed by a peak at 1635 which shows the presence of $-\mathrm{C}=\mathrm{O}$. The presence of marked peaks at 1630- 1650 and 2925- 2960 demonstrated the presence of PHA characteristics ester carbonyl bond. The FTIR results of pseudomonas B2 strain was in agreement with the earlier works (Wei et al., 2011; Muthazhagan and Thangaraj, 2014). The proton NMR spectra showed (Fig. 6) the characteristic peaks of PHBs near 5.25 ppm characteristic of the methyne group and PHBVs near $5.17 \mathrm{ppm}$. The spectral analysis suggested that considerable amount of PHV was produced by strain B2. Identical observations were reported by Bhuwal et al. (2014) in which they characterized chemical characterization of the extracted PHB by ${ }^{1} \mathrm{HNMR}$, Fourier transform infrared spectroscopy (FTIR), thermal gravimetric analysis (TGA), to determine the structure, melting point, and molecular mass of the purified PHB.

Poly hydroxyl butyrate miscibility analysis with polymer plastic and starch: As the PHB was found to be fragile and easily breakable, So its mechanical properties can be improved by blending PHB with other polymers like plastic and soluble starch. The polyhydroxybutyrate extracted in chloroform was mixed with equal weight of poly-propylene polymer plastic. The mixture was incubated at $70^{\circ} \mathrm{C}$ for $10 \mathrm{~min}$ utes and then poured into a glass petri plate. A film of blended mixture was obtained (Fig.7, A). The PHB blend sheet was prepared by mixing extracted polymer with soluble starch following the conventional solvent cast technique. Starch is used for blending because it has many benefit of being biodegradable, biocompatible, inexpensive, and also readily available while all other biodegradable polymer such as polyethylene glycol and polylactic acid are costly. Identical observations were reported by Choi et al. (2003) in which they prepared PHB sheet with EPB blends. The blends showed a higher thermal stability compared to PHB sheet. Bhuwal et al. (2014) reported the polymer sheet of extracted polymer prepared by blending the polymer with starch for packaging applications. This suggested at harmonious intermixing of PHB with the polymer plastic and starch. This in turn would lead us towards commercialization of blends towards marketable commodity.

Biodegradability analysis of agribioproducts: Biodegradation process of agro bioplastic was also studied under lab conditions (Fig. 7 B). For this purpose equal amount of agro-bioplastic was added to tubes containing sterile distilled water, soil water, sewage water and water containing bacterial culture. The process of degradation was studied till thirty days. It was observed that degradation process starts with in a week.

\section{Conclusion}

The medium composition was optimized by statistical design for bacterial for PHB recovery and PHB weight production by from Pseudomonas B2. The mathematical model generated from the $\mathrm{CCD}$ for $\mathrm{PHB}$ produc- 
tion and PHB weight had high correlation coefficient (R2), 0.84 and 0.91 respectively after screening and optimization by RSM. The optimized conditions included carbon source glucose and nitrogen sources mustard cake and yeast extract, with concentration of $3 \mathrm{~g} / \mathrm{l}, 1.8 \mathrm{~g} / \mathrm{l}$ and $0.8 \mathrm{~g} / \mathrm{l}$ respectively. Presesnce of PHB was confirmed by FTIR and NMR. Extracted PHB was checked for its miscibility with other polymers. In further investigation, the development of a mathematical model for batch fermentation and simulation of fed batch fermentation in a bioreactor should be done.

\section{REFERENCES}

Ahn, W,S., Park, S.J. and Lee, S.Y. (2001). Production of poly(3-hydroxybutyrate) from whey by cell recycle fedbatch culture of recombinant Escherichia coli. Biotechnol. Lett. 23:235-40.

Anderson, A.J. and Dawes, E.A. (1990). Occurrence, metabolism, metabolic role and industrial uses of bacterial polyhydroxyalkanoates. Microbiol. Rev. 54:450-72.

Beringer, J.F., Beynon. I.L., Buchanan-Wollaston, A.V. and Johnston, A.W.B.(1978). Transfer of the drug-resistance transposon Tn5 to Rhizobium. Nature. 276: 633-634.

Bhuwal, A.K., Singh, G., Aggarwal, N.K., Goyal, V. and Yadav, A. (2014). Poly- $\beta$-hydroxybutyrate production and management of cardboard industry effluent by new Bacillus sp. NA10. Bioresour. Bioprocess. 1:2-11.

Byrom, D. (1887). Polymer synthesis by microorganisms: technology and economics. Trends Biotechnol. 5:246-50.

Choi, J.Y., Lee, J.K., You, Y., Park, W.H. (2003). Epoxidized polybutadiene as a thermal stabilizer for poly(3hydroxybutyrate). II. Thermal stabilization of poly (3hydroxybutyrate) by epoxidized polybutadiene. Fiber Polym 4:195-198.

Jangra, M. and Sikka, V.K. (2015). Expeditious and ecofriendly production of poly- $\beta$-hydroxy butyrate in bacteria grown on renewable agribyproducts. Int. J. Recent Sci. Res. 6:6400-6404.

Jangra, M.R., Jain, A., Batra, R., Ahlawat, R. and Sikka, V.K. (2016). Stastical analysis for optimization of bacterial polyhydroxybutyrate production using agri byproducts. Indian J. Eco. 43:557-562.

Labuzeck, S. and Radecka, I. (2001). Biosynthesis of tercopolymer by Bacillus cereus UW85. J. Appl. Microbiol. 90:353-357.

Launen, L.A., Pinto, L.J. and Moore, M.M. (1999). Optimization of pyrene oxidation by Penicillium janthinellum using response surface methodology. Appl. Microbiol. Biotechnol. 51:510-5.

Lee, S.Y. (1996). Bacterial polyhydroxyalkanoates. Biotechnol. Bioeng. 49:1-14.

Montgomery, D.C. (2005). Design and analysis of experiments, 6th edn. Wiley, New York.

Muthazhagan, K. and Thangaraj, M. (2014). Production and ftir analysis of bio-polymer by bacillus sp isolated from vellar estuary sediment. Int. J. Sci. Invention Day. 3:625 -638 .

Sharma P and Bajaj BK (2015). Cost-effective-substrates for production of poly- $\beta$-hydroxybutyrate by a newly isolated Bacillus cereus PS-10. J. Environ. Biol. 36:1297-304.

Slepecky, R.A. and Law, J.H. (1961). Assay of poly- $\beta$ hydroxybutyric acid. J. Bacteriol. 82: 33-36.

Veeramanikandan, V., Mrudula, S., Yuvaraj, R. and Balaji, P. (2013). Optimization of Bacterial Poly $-\beta$ - Hydroxy Butyrate (PHB) Production from Different Industrial Waste using Central Composite Design. Int. J. Com. Appl. 62:27-34.

Wei, Y.H., Chen, W.C., Wu, H.S. and Janarthanan, O.M. (2011). Biodegradable and Biocompatible Biomaterial, Polyhydroxybutyrate, Produced by an Indigenous Vibrio sp. BM-1 Isolated from Marine Environment. Mar. Drugs. 9: 615-624. 